\title{
Existence of positive solutions for a class of fractional differential equations with the derivative term via a new fixed point theorem
}

\author{
Yanbin Sang ${ }^{1 *} \mathbb{D}$, Luxuan $\mathrm{He}^{1}$, Yanling Wang ${ }^{2}$, Yaqi $\mathrm{Ren}^{3}$ and $\mathrm{Na} \mathrm{Shi}^{1}$
}

\section{"Correspondence:}

syb6662004@163.com

'Department of Mathematics,

School of Science, North University

of China, Taiyuan, Shanxi, 030051

P.R. China

Full list of author information is

available at the end of the article

\begin{abstract}
In this paper, we firstly establish the existence and uniqueness of solutions of the operator equation $A(x, x)+B(x, x)+C(x)+e=x$, where $A$ and $B$ are two mixed monotone operators, $C$ is a decreasing operator, and $e \in P$ with $\theta \leq e \leq h$. Then, using our abstract theorem, we prove a class of fractional boundary value problems with the derivative term to have a unique solution and construct the corresponding iterative sequences to approximate the unique solution.
\end{abstract}

Keywords: Existence and uniqueness; Mixed monotone operator; Decreasing operator; Derivative term; Fractional equation

\section{Introduction}

In this paper, we consider the following fractional order boundary value problem:

$$
\left\{\begin{array}{l}
D_{0^{+}}^{\alpha} u(t)+f\left(t, u(t), D_{0^{+}}^{\beta} u(t)\right)+g(t, u(t),(H u)(t))-b=0, \quad t \in(0,1), \\
u(0)=u^{\prime}(0)=\cdots=u^{(n-2)}(0)=0, \\
{\left[D_{0^{+}}^{\gamma} u(t)\right]_{t=1}=k(u(1)),}
\end{array}\right.
$$

where $b>0$ is a constant, $n-1<\alpha \leq n, 1 \leq \beta<\gamma \leq n-2, n>3(n \in \mathbb{N}) . f, g:[0,1] \times$ $(-\infty,+\infty) \times(-\infty,+\infty) \rightarrow(-\infty,+\infty)$ are continuous functions, $k:[0,+\infty) \rightarrow[0,+\infty)$ is a continuous function, and $D_{0^{+}}^{\alpha}$ is the Riemann-Liouville fractional derivative of order $\alpha$.

Fractional differential equations have been increasingly adopted to describe some physical phenomena in thermology, electromagnetic wave, electrochemistry, and other applications [1-8]. There are a great deal of results about the existence and uniqueness of positive solutions for fractional boundary value problems. For example, Zhao and Gong [9] studied the unique positive solution of a class of higher order fractional equations with a parameter by Banach fixed point theorem. In [10], Wang, Zhang, and Wang obtained fixed point theorems of nonlinear sum operators and applications in a fractional differential equation. On the other hand, much attention has been paid to fractional differential equations involving nonlinearities with the derivative term. In [11], Ji et al. investigated positive solutions for the nonlinear fractional differential equation with a derivative term.

(c) The Author(s) 2021. This article is licensed under a Creative Commons Attribution 4.0 International License, which permits use, sharing, adaptation, distribution and reproduction in any medium or format, as long as you give appropriate credit to the original author(s) and the source, provide a link to the Creative Commons licence, and indicate if changes were made. The images or other third party material in this article are included in the article's Creative Commons licence, unless indicated otherwise in a credit line to the material. If material is not included in the article's Creative Commons licence and your intended use is not permitted by statutory regulation or exceeds the permitted use, you will need to obtain permission directly from the copyright holder. To view a copy of this licence, visit http://creativecommons.org/licenses/by/4.0/. 
Moreover, Yue and Zou [12] were concerned with a class of fractional Dirichlet boundary value problems with dependence on the first order derivative. Some sufficient conditions for the uniqueness of solutions for the above-mentioned problem were given. The main tool is also classical Banach's contraction mapping principle.

It is well known that problem (1.1) is the generalization of elastic beam equation [13]. In [14], Goodrich first studied the Green's function associated with problem (1.1) when $k \equiv 0$ and established the existence result on sublinear nonlinearity. Furthermore, $\mathrm{Xu}$, Wei, and Dong [15] also considered sublinear problem (1.1) by using of the fixed point index theorem and spectral theory. Jleli and Samet [16] utilized a mixed monotone fixed point theorem to obtain a unique solution of problem (1.1) when $b=0$. Moreover, Yang, Shen, and Xie [17] investigated the nonlinear term involving the first order derivative for problem (1.1).

We should mention the main results obtained in [18-22], which motivated us to consider problem (1.1). In [18], Wang and Zhang studied the operator equation $A x+B x+$ $C(x, x)=x$, where $A$ is an increasing $\alpha$-concave operator, $B$ is a decreasing operator, and $C$ is a mixed monotone operator. Existence and uniqueness of the operator equation were established. Furthermore, Zhang and Tian [19] considered the following fractional boundary value problem:

$$
\left\{\begin{array}{l}
D_{0^{+}}^{\alpha} x(t)+f\left(t, x(t), D_{0^{+}}^{\beta} x(t)\right)+g(t, x(t))=0, \quad t \in(0,1) \\
x(0)=x^{\prime}(0)=\cdots=x^{(n-2)}(0)=0 \\
{\left[D_{0^{+}}^{\gamma} x(t)\right]_{t=1}=k(x(1))}
\end{array}\right.
$$

where $n \geq 3,1 \leq \beta \leq \gamma \leq n-2, f:[0,1] \times[0,+\infty) \times[0,+\infty) \longrightarrow[0,+\infty), g:[0,1] \times$ $[0,+\infty) \longrightarrow[0,+\infty)$, and $k:[0,+\infty) \longrightarrow[0,+\infty)$. The authors used the abstract theorem obtained in [18] to prove that problem (1.2) admits a unique positive solution. Subsequently, Wang [20] considered the following singular nonlinear fractional differential equation:

$$
\left\{\begin{array}{l}
D_{0^{+}}^{\alpha} u(t)+p(t) f\left(t, u(t), D_{0^{+}}^{\beta} u(t)\right)+q(t) g(t, u(t),(H u)(t))=0, \quad 0<t<1, \\
u(0)=u^{\prime}(0)=\cdots=u^{(n-2)}(0)=0, \\
{\left[D_{0^{+}}^{\gamma} u(t)\right]_{t=1}=k(u(1)),}
\end{array}\right.
$$

where $n-1<\alpha \leq n, n>3,1 \leq \beta \leq \gamma \leq n-2, p, q \in C((0,1),[0,+\infty)), p(t)$ and $q(t)$ are allowed to be singular at $t=0$ or $t=1$. $f:(0,1) \times(0,+\infty) \times(0,+\infty) \longrightarrow[0,+\infty)$ is continuous, $g:(0,1) \times[0,+\infty) \times[0,+\infty) \longrightarrow[0,+\infty)$ is continuous, and $k:[0,1) \rightarrow[0,+\infty)$ is also continuous. The author proved problem (1.3) to have a unique positive solution based on a new mixed monotone fixed theorem. Very recently, Sang and Ren [21] investigated the following fractional boundary value problem:

$$
\left\{\begin{array}{l}
-D_{0^{+}}^{\alpha} u(t)=f(t, u(t), u(t))+g(t, u(t), u(t))-b, \quad 0<t<1, n-1<\alpha \leq n \\
u^{(i)}(0)=0, \quad 0 \leq i \leq n-2 \\
{\left[D_{0^{+}}^{\beta} u(t)\right]_{t=1}=0, \quad 1 \leq \beta \leq n-2}
\end{array}\right.
$$


where $n \geq 3(n \in \mathbb{N}), b>0$ is a constant, $f, g:[0,1] \times(-\infty,+\infty) \times(-\infty,+\infty) \rightarrow(-\infty,+\infty)$ are continuous functions. In fact, Zhai and Wang [22] considered the following problem:

$$
\left\{\begin{array}{l}
D_{0^{+}}^{\alpha} u(t)+f(t, u(t))=b, \quad t \in[0,1] \\
u(0)=u^{\prime}(0)=0 \\
u(1)=\beta \int_{0}^{1} u(s) d s
\end{array}\right.
$$

where $2<\alpha \leq 3,0<\beta<\alpha, b>0$ is a constant, $f:[0,1] \times(-\infty,+\infty) \longrightarrow(-\infty,+\infty)$ is continuous. The authors introduced $\phi-(h, e)$ operators and used monotone iterative method to establish the existence and uniqueness of a nontrivial solution for problem (1.5).

Compared with problem (1.4), we add the derivative term $D_{0^{+}}^{\beta} u(t)$, the operator term $(H u)(t)$, and nonlinear boundary conditions $k(u(1))$ into problem (1.1). Furthermore, different from problems (1.2) and (1.3), we break through the restriction of positivity on nonlinearities $f$ and $g$. The first goal of this paper is to establish the existence and uniqueness theorem of solution for the operator equation $A(x, x)+B(x, x)+C(x)+e=x$, where $A$ and $B$ are both mixed monotone, $C(x)$ is decreasing, and $e \in P$ with $P$ is a cone in Banach space $E$. Our abstract theorem generalizes the result on the cone mappings (see Theorem 3.1 in [19]) to non-cone case. Some sufficient conditions under which problem (1.1) has a unique solution are provided. Moreover, we also construct two iterative sequences for approximating a unique solution.

The structure of this paper includes the following sections. In Sect. 2, we introduce some definitions and give preliminary results to be used in the proof of our main theorems. In Sect. 3, we establish the existence and uniqueness of solutions for problem (1.1) based on a new fixed point theorem.

\section{Preliminaries}

In this section, we give some definitions and preliminary results that are used in this paper [23-25].

In this paper, $(E,\|\cdot\|)$ is a real Banach space, which is partially ordered by a cone $P \subset E$, i.e., $x \leq y$ if and only if $y-x \in P . \theta$ is the zero element in $E$. Recall that a nonempty closed convex set $P \subset E$ is a cone if it satisfies: $x \in P, \lambda \geq 0 \Rightarrow \lambda x \in P$ and $x \in P,-x \in P \Rightarrow x=\theta$. $P$ is called to be normal if there exists $N>0$ such that $\theta \leq x \leq y \Rightarrow\|x\| \leq N\|y\|$. Given $h>\theta$, we denote $P_{h}$ by

$$
P_{h}=\{x \in E \mid \text { there exist } \lambda>0, \mu>0 \text { such that } \lambda h \leq x \leq \mu h\}
$$

Let $e \in P$ with $\theta \leq e \leq h$, we define

$$
P_{h, e}=\left\{x \in E \mid x+e \in P_{h}\right\} .
$$

Definition 2.1 ([23, 24]) Let an operator $A: P_{h, e} \times P_{h, e} \rightarrow E$ be a mixed monotone operator if $A(x, y)$ is increasing in $x$ and decreasing in $y$, i.e., for $u_{i}, v_{i} \in P_{h, e},(i=1,2), u_{1} \leq v_{1}$, $v_{2} \leq u_{2}$ imply $A\left(u_{1}, u_{2}\right) \leq A\left(v_{1}, v_{2}\right)$. The element $x \in P_{h, e}$ is called a fixed point of $A$ if $A(x, x)=x$.

Lemma 2.1 ([21]) Let $P$ be a normal cone of $E$ and $T: P_{h, e} \times P_{h, e} \longrightarrow$ E be a mixed monotone operator with $T(h, h) \in P_{h, e}$, and the following condition is satisfied: 
(H) There exists a mapping $\varphi:(0,1) \longrightarrow(0,+\infty)$ with $\varphi(\lambda)>\lambda$ such that

$$
T\left(\lambda u+(\lambda-1) e, \lambda^{-1} v+\left(\lambda^{-1}-1\right) e\right) \geq \varphi(\lambda) T(u, v)+(\varphi(\lambda)-1) e
$$

for all $u, v \in P_{h, e}$ and $\lambda \in(0,1)$. Then

(1) There exist $u_{0}, v_{0} \in P_{h, e}$ and $s \in(0,1)$ such that

$$
s v_{0} \leq u_{0}<v_{0}, \quad u_{0} \leq T\left(u_{0}, v_{0}\right) \leq T\left(v_{0}, u_{0}\right) \leq v_{0}
$$

(2) Thas a unique fixed point $x^{*}$ in $P_{h, e}$;

(3) For any initial values $x_{0}, y_{0} \in P_{h, e}$, by constructing successively the sequence as follows:

$$
x_{n}=T\left(x_{n-1}, y_{n-1}\right), \quad y_{n}=T\left(y_{n-1}, x_{n-1}\right), \quad n=1,2, \ldots,
$$

we have $x_{n} \rightarrow x^{*}$ and $y_{n} \rightarrow x^{*}$ as $n \rightarrow \infty$.

Definition 2.2 ([3]) The Riemann-Liouville fractional derivative of order $\alpha$ of a function $y \in C[0,1]$ is defined by

$$
D_{0^{+}}^{\alpha} y(t)=\frac{1}{\Gamma(n-\alpha)}\left(\frac{d}{d t}\right)^{n} \int_{0}^{t} \frac{y(s)}{(t-s)^{\alpha-n-1}} d s
$$

where $n=[\alpha]+1,[\alpha]$ denotes the integer part of the number $\alpha$, provided that the righthand side is pointwise defined on $(0, \infty)$.

Lemma $2.2([19,26])$ Let $h(t) \in C[0,1]$, then the unique solution of the linear problem

$$
\left\{\begin{array}{l}
D_{0^{+}}^{\alpha} u(t)+h(t)=0, \quad 0<t<1, n-1<\alpha \leq n \\
u(0)=u^{\prime}(0)=\cdots=u^{(n-2)}(0)=0 \\
{\left[D_{0^{+}}^{\gamma} u(t)\right]_{t=1}=k(u(1)), \quad 1 \leq \gamma \leq n-2,}
\end{array}\right.
$$

is given by

$$
u(t)=\int_{0}^{1} G(t, s) h(s) d s+\frac{\Gamma(\alpha-\gamma)}{\Gamma(\alpha)} k(u(1)) t^{\alpha-1}
$$

where

$$
G(t, s)=\frac{1}{\Gamma(\alpha)} \begin{cases}t^{\alpha-1}(1-s)^{\alpha-\gamma-1}-(t-s)^{\alpha-1}, & 0 \leq s \leq t \leq 1 \\ t^{\alpha-1}(1-s)^{\alpha-\gamma-1}, & 0 \leq t \leq s \leq 1\end{cases}
$$

is the Green's function.

Lemma 2.3 ([19]) The Green's function $G(t, s)$ in Lemma 2.2 has the following properties:

(1) $G(t, s):[0,1] \times[0,1] \rightarrow[0, \infty)$ is continuous; 
(2) For all $t, s \in[0,1]$, we have

$$
0 \leq\left[1-(1-s)^{\gamma}\right](1-s)^{\alpha-\gamma-1} t^{\alpha-1} \leq \Gamma(\alpha) G(t, s) \leq(1-s)^{\alpha-\gamma-1} t^{\alpha-1}
$$

(3) For all $t, s \in[0,1]$, we have

$$
0 \leq\left[1-(1-s)^{\gamma-\beta}\right](1-s)^{\alpha-\gamma-1} t^{\alpha-\beta-1} \leq \Gamma(\alpha-\beta) D_{0^{+}}^{\beta} G(t, s) \leq(1-s)^{\alpha-\gamma-1} t^{\alpha-\beta-1} .
$$

Theorem 2.1 Let $P$ be a normal cone in $E$, and let $A, B: P_{h, e} \times P_{h, e} \rightarrow E$ be two mixed monotone operators, $C: P \rightarrow P$ be a decreasing operator satisfying the following conditions:

(A1) For all $t \in(0,1)$ and $x, y \in P_{h, e}$, there exists $\psi(t) \in(t, 1)$ such that

$$
A\left(t x+(t-1) e, t^{-1} y+\left(t^{-1}-1\right) e\right) \geq \psi(t) A(x, y)+(\psi(t)-1) e
$$

(A2) For all $t \in(0,1)$ and $x, y \in P_{h, e}$,

$$
B\left(t x+(t-1) e, t^{-1} y+\left(t^{-1}-1\right) e\right) \geq t B(x, y)+(t-1) e
$$

(A3) For all $t \in(0,1)$ and $y \in P$, we have

$$
C\left(t^{-1} y+\left(t^{-1}-1\right) e\right) \geq t C(y)
$$

(A4) $A(h, h) \in P_{h, e}, B(h, h) \in P_{h, e}$, and $C(h) \in P_{h}$;

(A5) For all $x, y \in P_{h, e}$, there exists a constant $\delta>0$ such that

$$
A(x, y) \geq \delta(B(x, y)+C(y))+(\delta-1) e .
$$

Then the operator equation $A(x, x)+B(x, x)+C(x)+e=x$ has a unique solution $x^{*}$ in $P_{h, e}$, and for any initial values $x_{0}, y_{0} \in P_{h, e}$, by setting two iterative sequences $\left\{x_{n}\right\}\left\{y_{n}\right\}$ as follows:

$$
\begin{aligned}
& x_{n}=A\left(x_{n-1}, y_{n-1}\right)+B\left(x_{n-1}, y_{n-1}\right)+C\left(y_{n-1}\right)+e, \quad n=1,2, \ldots, \\
& y_{n}=A\left(y_{n-1}, x_{n-1}\right)+B\left(y_{n-1}, x_{n-1}\right)+C\left(x_{n-1}\right)+e, \quad n=1,2, \ldots,
\end{aligned}
$$

we have $x_{n} \rightarrow x^{*}$ and $y_{n} \rightarrow x^{*}$ in $E$ as $n \rightarrow \infty$.

Proof We prove Theorem 2.1 in view of Lemma 2.1. Firstly, by condition (A4), and combining with Lemma 2.2 in [18], we have that there exist constants $a_{i}>0$ and $b_{i}>0$ $(i=1,2,3)$ such that

$$
\begin{aligned}
& a_{1} h+\left(a_{1}-1\right) e \leq A(h, h) \leq b_{1} h+\left(b_{1}-1\right) e, \\
& a_{2} h+\left(a_{2}-1\right) e \leq B(h, h) \leq b_{2} h+\left(b_{2}-1\right) e, \\
& a_{3} h \leq C(h) \leq b_{3} h .
\end{aligned}
$$


Consequently, for all $x, y \in P_{h, e}$, by [21], we obtain

$$
\psi(\mu) a_{1} h+\left(\psi(\mu) a_{1}-1\right) e \leq A(x, y) \leq \psi(\mu)^{-1} b_{1} h+\left(\psi(\mu)^{-1} b_{1}-1\right) e, \quad \mu \in(0,1)
$$

Hence $A(x, y) \in P_{h, e}$, that is, $A: P_{h, e} \times P_{h, e} \rightarrow P_{h, e}$. Similarly, for all $x, y \in P_{h, e}$, we deduce that

$$
\eta a_{2} h+\left(\eta a_{2}-1\right) e \leq B(x, y) \leq \eta^{-1} b_{2} h+\left(\eta^{-1} b_{2}-1\right) e, \quad \eta \in(0,1) .
$$

Therefore $B: P_{h, e} \times P_{h, e} \rightarrow P_{h, e}$.

For all $y \in P_{h}$, there exists $\sigma \in(0,1)$ such that $\sigma h \leq y \leq \sigma^{-1} h$. Since $C$ is a decreasing operator, we have

$$
\begin{aligned}
& C(y) \geq C\left(\sigma^{-1} h\right) \geq \sigma C(h) \geq \sigma a_{3} h, \\
& C(y) \leq C(\sigma h) \leq \sigma^{-1} C(h) \leq \sigma^{-1} b_{3} h .
\end{aligned}
$$

Let $m_{1}=\sigma a_{3}, m_{2}=\sigma^{-1} b_{3}$, that is, $m_{1} h \leq C(y) \leq m_{2} h$. Hence $C(y) \in P_{h}$, that is, $C: P_{h} \rightarrow$ $P_{h}$.

Now we define the operator $T=A+B+C+e: P_{h, e} \times P_{h, e} \rightarrow E$ by

$$
T(x, y)=A(x, y)+B(x, y)+C(y)+e \quad \text { for all } x, y \in P_{h, e} .
$$

Let $x_{i}, y_{i} \in P_{h, e}(i=1,2)$ with $x_{1} \leq x_{2}, y_{1} \geq y_{2}$, we obtain

$$
A\left(x_{1}, y_{1}\right) \leq A\left(x_{2}, y_{2}\right), \quad B\left(x_{1}, y_{1}\right) \leq B\left(x_{2}, y_{2}\right), \quad C\left(y_{1}\right) \leq C\left(y_{2}\right) .
$$

Hence, $T\left(x_{1}, y_{1}\right) \leq T\left(x_{2}, y_{2}\right)$, $\mathrm{T}$ is a mixed monotone operator.

From (2.4), we have

$$
T(h, h)=A(h, h)+B(h, h)+C(h)+e
$$

By (2.1)-(2.3), we can deduce that

$$
T(h, h) \geq\left(a_{1}+a_{2}+a_{3}\right) h+\left(a_{1}+a_{2}-1\right) e \geq\left(a_{1}+a_{2}\right) h+\left(a_{1}+a_{2}-1\right) e
$$

and

$$
T(h, h) \leq\left(b_{1}+b_{2}+b_{3}\right) h+\left(b_{1}+b_{2}-1\right) e \leq\left(b_{1}+b_{2}+b_{3}\right) h+\left(b_{1}+b_{2}+b_{3}-1\right) e .
$$

Let $\varphi_{1}=a_{1}+a_{2}$ and $\varphi_{2}=b_{1}+b_{2}+b_{3}$. Then

$$
\varphi_{1} h+\left(\varphi_{1}-1\right) e \leq T(h, h) \leq \varphi_{2} h+\left(\varphi_{2}-1\right) e
$$

Hence $T(h, h) \in P_{h, e}$. 
Finally, we prove that, for every $t \in(0,1)$, there exists $\varphi(t) \in(t, 1]$ such that, for all $x, y \in$ $P_{h, e}$,

$$
T\left(t x+(t-1) e, t^{-1} y+\left(t^{-1}-1\right) e\right) \geq \varphi(t) T(x, y)+(\varphi(t)-1) e .
$$

By condition (A5), we have that

$$
A(x, y)+\delta A(x, y) \geq \delta(B(x, y)+C(y))+(\delta-1) e+\delta A(x, y),
$$

that is,

$$
A(x, y) \geq \frac{\delta}{1+\delta} T(x, y)-\frac{e}{1+\delta} .
$$

Moreover, it follows from (2.4), (2.5) and conditions (A1)-(A3) that

$$
\begin{aligned}
T( & t x \\
+ & \left.(t-1) e, t^{-1} y+\left(t^{-1}-1\right) e\right)-t T(x, y) \\
= & A\left(t x+(t-1) e, t^{-1} y+\left(t^{-1}-1\right) e\right)+B\left(t x+(t-1) e, t^{-1} y+\left(t^{-1}-1\right) e\right) \\
& +C\left(t^{-1} y+\left(t^{-1}-1\right) e\right)+e-t(A(x, y)+B(x, y)+C(y)+e) \\
\geq & \psi(t) A(x, y)+(\psi(t)-1) e+t B(x, y)+(t-1) e+t C(y)+e-t A(x, y) \\
& -t B(x, y)-t C(y)-t e \\
= & (\psi(t)-t) A(x, y)+(\psi(t)-1) e \\
\geq & (\psi(t)-t)\left(\frac{\delta}{1+\delta} T(x, y)-\frac{e}{1+\delta}\right)+(\psi(t)-1) e \\
= & \frac{\delta(\psi(t)-t)}{1+\delta} T(x, y)+\left(\psi(t)-1-\frac{\psi(t)-t}{1+\delta}\right) e \quad \text { for all } x, y \in P_{h, e} .
\end{aligned}
$$

Thus

$$
\begin{aligned}
& T\left(t x+(t-1) e, t^{-1} y+\left(t^{-1}-1\right) e\right) \\
& \quad \geq\left(\frac{\delta(\psi(t)-t)}{1+\delta}+t\right) T(x, y)+\left(\psi(t)-1-\frac{\psi(t)-t}{1+\delta}\right) e \\
& \quad=\frac{\delta \psi(t)+t}{1+\delta} T(x, y)+\left(\frac{\delta \psi(t)+t}{1+\delta}-1\right) e \quad \text { for } x, y \in P_{h, e} .
\end{aligned}
$$

Let $\varphi(t)=\frac{\delta \psi(t)+t}{1+\delta}$, then $\varphi(t) \in(t, \psi(t)) \subset(t, 1], t \in(0,1)$, by (2.6), we can conclude that

$$
T\left(t x+(t-1) e, t^{-1} y+\left(t^{-1}-1\right) e\right) \geq \varphi(t) T(x, y)+(\varphi(t)-1) e, \quad \forall x, y \in P_{h, e} .
$$

We derive the conclusion of Theorem 2.1 from Lemma 2.1.

\section{Main result}

In the section, we use Theorem 2.1 to obtain the existence and uniqueness of a positive solution for problem (1.1).

Set $E=\left\{x \mid x \in C[0,1], D_{0^{+}}^{\beta} x \in C[0,1]\right\}$, then $E$ is a Banach space with an order relation $u \leq$ $v$ if $u(t) \leq v(t), D_{0^{+}}^{\beta} u(t) \leq D_{0^{+}}^{\beta} v(t)$. Let $P \subset E$ be defined by $P=\left\{x \in E \mid x(t) \geq 0, D_{0^{+}}^{\beta} x(t) \geq 0\right\}$ 
for all $t \in[0,1]$. It is clear that $P$ is a normal cone. Let

$$
e(t)=\frac{b}{(\alpha-\gamma) \Gamma(\alpha)}\left(t^{\alpha-1}-\frac{\alpha-\gamma}{\alpha} t^{\alpha}\right), \quad t \in[0,1] .
$$

Theorem 3.1 Assume that the following conditions are satisfied:

(B1) $f, g:[0,1] \times\left[-e^{*},+\infty\right) \times\left[-e^{*},+\infty\right) \rightarrow(-\infty,+\infty)$ are continuous and $k:[0,+\infty) \rightarrow[0,+\infty)$ is continuous. For all $t \in[0,1], g(t, 0, H(L)) \geq 0$ with $g(t, 0, H(L)) \not \equiv 0$, where $L \geq \frac{b}{(\alpha-\gamma) \Gamma(\alpha)}$ and $e^{*}=\max \{e(t): t \in[0,1]\}$;

(B2) For fixed $t \in[0,1]$ and $y \in\left[-e^{*},+\infty\right), f(t, x, y), g(t, x, y)$ are increasing in $x \in\left[-e^{*},+\infty\right)$; for fixed $t \in[0,1]$ and $x \in\left[-e^{*},+\infty\right), f(t, x, y), g(t, x, y)$ are decreasing in $y \in\left[-e^{*},+\infty\right) ; k(y)$ is decreasing in $y \in[0,+\infty), k(L) \neq 0$;

(B3) For all $\lambda \in(0,1)$, there exists $\psi(\lambda) \in(\lambda, 1)$ such that, for all $t \in[0,1]$,

(a) $f\left(t, \lambda x+(\lambda-1) \rho_{1}, \lambda^{-1} y+\left(\lambda^{-1}-1\right) \rho_{2}\right) \geq \psi(\lambda) f(t, x, y)$,

(b) $g\left(t, \lambda x+(\lambda-1) \rho_{1}, \lambda^{-1} y+\left(\lambda^{-1}-1\right) \rho_{2}\right) \geq \lambda g(t, x, y)$,

(c) $k\left(\lambda^{-1} y+\left(\lambda^{-1}-1\right) \rho_{1}\right) \geq \lambda k(y)$,

where $x, y \in(-\infty,+\infty)$ and $\rho_{1}, \rho_{2} \in\left[0, e^{*}\right]$;

(B4) For all $t \in[0,1], x, y \in\left[-e^{*},+\infty\right)$, there exist two constants $\delta_{1}, \delta_{2}>0$ such that

(a) $f(t, x, y) \geq \delta_{1} g(t, x, 0)$,

(b) $f(t, x, y) \geq \delta_{2} k(y)$;

(B5) $H: C[0,1] \rightarrow C[0,1]$ and satisfies the following conditions:

(a) $H u \geq 0$ for every $u \in P_{h, e}$;

(b) for $u, v \in P_{h, e}, u \leq v \Longrightarrow H u \leq H v$;

(c) for all $\lambda \in(0,1)$ and $u \in P_{h, e}$ such that

$$
H(\lambda u+(\lambda-1) \hat{e}) \geq \lambda H(u)+(\lambda-1) \hat{e}, \quad \hat{e} \in\left[0, e^{*}\right]
$$

Then we have the following conclusions:

(1) Problem (1.1) has a unique nontrivial solution $u^{*}$ in $P_{h, e}$, where $h(t)=L t^{\alpha-1}$ for all $t \in[0,1]$

(2) We can construct the following two sequences:

$$
\begin{aligned}
\omega_{n}(t)= & \int_{0}^{1} G(t, s)\left[f\left(s, \omega_{n-1}(s), D_{0^{+}}^{\beta} \tau_{n-1}(s)\right)+g\left(s, \omega_{n-1}(s),\left(H \tau_{n-1}\right)(s)\right)\right] d s \\
& +\frac{\Gamma(\alpha-\gamma)}{\Gamma(\alpha)} k\left(\tau_{n-1}(1)\right) t^{\alpha-1}-e(t), \quad n=1,2, \ldots, \\
\tau_{n}(t)= & \int_{0}^{1} G(t, s)\left[f\left(s, \tau_{n-1}(s), D_{0^{+}}^{\beta} \omega_{n-1}(s)\right)+g\left(s, \tau_{n-1}(s),\left(H \omega_{n-1}\right)(s)\right)\right] d s \\
& +\frac{\Gamma(\alpha-\gamma)}{\Gamma(\alpha)} k\left(\omega_{n-1}(1)\right) t^{\alpha-1}-e(t), \quad n=1,2, \ldots,
\end{aligned}
$$

for any initial values $\omega_{0}, \tau_{0} \in P_{h, e}$, and sequences $\left\{\omega_{n}(t)\right\}$ and $\left\{\tau_{n}(t)\right\}$ for approximating $u^{*}(t)$, we have $\omega_{n}(t) \rightarrow u^{*}(t)$ and $\tau_{n}(t) \rightarrow u^{*}(t)$ as $n \rightarrow \infty$.

Proof We will use Theorem 2.1 to prove Theorem 3.1.

For $e \in P, t \in[0,1]$, we have

$$
P_{h, e}=\left\{x \in C[0,1] \mid x+e \in P_{h}\right\} .
$$


Furthermore, for $L \geq \frac{b}{(\alpha-\gamma) \Gamma(\alpha)}$ and $t \in[0,1]$, we have

$$
e(t)=\frac{b}{(\alpha-\gamma) \Gamma(\alpha)} t^{\alpha-1}-\frac{b}{\alpha \Gamma(\alpha)} t^{\alpha} \leq \frac{b}{(\alpha-\gamma) \Gamma(\alpha)} t^{\alpha-1} \leq L t^{\alpha-1}=h(t) .
$$

Hence $0<e(t) \leq h(t)$.

From Lemma 2.2, problem (1.1) has the integral formulation

$$
\begin{aligned}
u(t)= & \int_{0}^{1} G(t, s)\left[f\left(s, u(s), D_{0^{+}}^{\beta} u(s)\right)+g(s, u(s),(H u)(s))-b\right] d s \\
& +\frac{\Gamma(\alpha-\gamma)}{\Gamma(\alpha)} k(u(1)) t^{\alpha-1} \\
= & \int_{0}^{1} G(t, s) f\left(s, u(s), D_{0^{+}}^{\beta} u(s)\right) d s+\int_{0}^{1} G(t, s) g(s, u(s),(H u)(s)) d s \\
& +\frac{\Gamma(\alpha-\gamma)}{\Gamma(\alpha)} k(u(1)) t^{\alpha-1}-b \int_{0}^{1} G(t, s) d s \\
= & \int_{0}^{1} G(t, s) f\left(s, u(s), D_{0^{+}}^{\beta} u(s)\right) d s+\int_{0}^{1} G(t, s) g(s, u(s),(H u)(s)) d s \\
& +\frac{\Gamma(\alpha-\gamma)}{\Gamma(\alpha)} k(u(1)) t^{\alpha-1}-\frac{b}{(\alpha-\gamma) \Gamma(\alpha)}\left(t^{\alpha-1}-\frac{\alpha-\gamma}{\alpha} t^{\alpha}\right) \\
= & \int_{0}^{1} G(t, s) f\left(s, u(s), D_{0^{+}}^{\beta} u(s)\right) d s+\int_{0}^{1} G(t, s) g(s, u(s),(H u)(s)) d s \\
& +\frac{\Gamma(\alpha-\gamma)}{\Gamma(\alpha)} k(u(1)) t^{\alpha-1}-e(t) \\
= & \int_{0}^{1} G(t, s) f\left(s, u(s), D_{0^{+}}^{\beta} u(s)\right) d s-e(t)+\int_{0}^{1} G(t, s) g(s, u(s),(H u)(s)) d s \\
& -e(t)+\frac{\Gamma(\alpha-\gamma)}{\Gamma(\alpha)} k(u(1)) t^{\alpha-1}+e(t) .
\end{aligned}
$$

For every $t \in[0,1]$ and $u, v \in P_{h, e}$, we consider the following operators:

$$
\begin{aligned}
& A(u, v)(t)=\int_{0}^{1} G(t, s) f\left(s, u(s), D_{0^{+}}^{\beta} v(s)\right) d s-e(t), \\
& B(u, v)(t)=\int_{0}^{1} G(t, s) g(s, u(s),(H v)(s)) d s-e(t),
\end{aligned}
$$

and

$$
C(v)(t)=\frac{\Gamma(\alpha-\gamma)}{\Gamma(\alpha)} k(v(1)) t^{\alpha-1}
$$

It is clear that $u(t)$ is the solution of problem (1.1) if and only if $u$ is the fixed point of the operator $A(u, u)+B(u, u)+C(u)+e$. Further, by (3.2)-(3.4), we can calculate that

$$
\begin{aligned}
& D_{0^{+}}^{\beta} A(u, v)(t)=\int_{0}^{1} D_{0^{+}}^{\beta} G(t, s) f\left(s, u(s), D_{0^{+}}^{\beta} v(s)\right) d s-D_{0^{+}}^{\beta} e(t), \\
& D_{0^{+}}^{\beta} B(u, v)(t)=\int_{0}^{1} D_{0^{+}}^{\beta} G(t, s) g(s, u(s),(H v)(s)) d s-D_{0^{+}}^{\beta} e(t),
\end{aligned}
$$


and

$$
D_{0^{+}}^{\beta} C(v)(t)=\frac{\Gamma(\alpha-\gamma)}{\Gamma(\alpha-\beta)} k(v(1)) t^{\alpha-\beta-1} .
$$

(1) Firstly, for all $u_{i}, v_{i} \in P_{h, e}(i=1,2)$ with $u_{1} \geq u_{2}, v_{1} \leq v_{2}$, by (B5), we get that $H\left(v_{1}\right) \leq$ $H\left(v_{2}\right)$. It follows from condition (B2), (3.2), and (3.5) that

$$
\begin{aligned}
A\left(u_{1}, v_{1}\right)(t) & =\int_{0}^{1} G(t, s) f\left(s, u_{1}(s), D_{0^{+}}^{\beta} v_{1}(s)\right) d s-e(t) \\
& \geq \int_{0}^{1} G(t, s) f\left(s, u_{2}(s), D_{0^{+}}^{\beta} v_{2}(s)\right) d s-e(t)=A\left(u_{2}, v_{2}\right)(t),
\end{aligned}
$$

and

$$
\begin{aligned}
D_{0^{+}}^{\beta} A\left(u_{1}, v_{1}\right)(t) & =\int_{0}^{1} D_{0^{+}}^{\beta} G(t, s) f\left(s, u_{1}(s), D_{0^{+}}^{\beta} v_{1}(s)\right) d s-D_{0^{+}}^{\beta} e(t) \\
& \geq \int_{0}^{1} D_{0^{+}}^{\beta} G(t, s) f\left(s, u_{2}(s), D_{0^{+}}^{\beta} v_{2}(s)\right) d s-D_{0^{+}}^{\beta} e(t)=D_{0^{+}}^{\beta} A\left(u_{2}, v_{2}\right)(t) .
\end{aligned}
$$

Thus, $A$ is a mixed monotone operator. Similarly, we have from (3.3) and (3.6) that

$$
\begin{aligned}
B\left(u_{1}, v_{1}\right)(t) & =\int_{0}^{1} G(t, s) g\left(s, u_{1}(s),\left(H v_{1}\right)(s)\right) d s-e(t) \\
& \geq \int_{0}^{1} G(t, s) g\left(s, u_{2}(s),\left(H v_{2}\right)(s)\right) d s-e(t)=B\left(u_{2}, v_{2}\right)(t)
\end{aligned}
$$

and

$$
\begin{aligned}
D_{0^{+}}^{\beta} B\left(u_{1}, v_{1}\right)(t) & =\int_{0}^{1} D_{0^{+}}^{\beta} G(t, s) g\left(s, u_{1}(s),\left(H v_{1}\right)(s)\right) d s-D_{0^{+}}^{\beta} e(t) \\
& \geq \int_{0}^{1} D_{0^{+}}^{\beta} G(t, s) g\left(s, u_{2}(s),\left(H v_{2}\right)(s)\right) d s-D_{0^{+}}^{\beta} e(t)=D_{0^{+}}^{\beta} B\left(u_{2}, v_{2}\right)(t) .
\end{aligned}
$$

Hence, $B$ is a mixed monotone operator. Since

$$
C\left(v_{1}\right)(t)=\frac{\Gamma(\alpha-\gamma)}{\Gamma(\alpha)} k\left(v_{1}(1)\right) t^{\alpha-1} \geq \frac{\Gamma(\alpha-\gamma)}{\Gamma(\alpha)} k\left(v_{2}(1)\right) t^{\alpha-1}=C\left(v_{2}\right)(t)
$$

and

$$
D_{0^{+}}^{\beta} C\left(v_{1}\right)(t)=\frac{\Gamma(\alpha-\gamma)}{\Gamma(\alpha-\beta)} k\left(v_{1}(1)\right) t^{\alpha-\beta-1} \geq \frac{\Gamma(\alpha-\gamma)}{\Gamma(\alpha-\beta)} k\left(v_{2}(1)\right) t^{\alpha-\beta-1}=D_{0^{+}}^{\beta} C\left(v_{2}\right)(t),
$$

Then $C$ is a decreasing operator.

(2) In view of condition (B3)(a), for every $\lambda \in[0,1]$ and $t \in[0,1]$, there exists $\psi(\lambda) \in(\lambda, 1)$ such that, for all $u, v \in P_{h, e}$, we have

$$
\begin{aligned}
A & \left(\lambda u+(\lambda-1) e, \lambda^{-1} v+\left(\lambda^{-1}-1\right) e\right)(t) \\
\quad & =\int_{0}^{1} G(t, s) f\left(s, \lambda u(s)+(\lambda-1) e, D_{0^{+}}^{\beta}\left(\lambda^{-1} v(s)+\left(\lambda^{-1}-1\right) e\right)\right) d s-e(t)
\end{aligned}
$$




$$
\begin{aligned}
& =\int_{0}^{1} G(t, s) f\left(s, \lambda u(s)+(\lambda-1) e, \lambda^{-1} D_{0^{+}}^{\beta} \nu(s)+\left(\lambda^{-1}-1\right) D_{0^{+}}^{\beta} e\right) d s-e(t) \\
& \geq \psi(\lambda) \int_{0}^{1} G(t, s) f\left(s, u(s), D_{0^{+}}^{\beta} \nu(s)\right) d s-e(t)+\psi(\lambda) e(t)-\psi(\lambda) e(t) \\
& =\psi(\lambda) A(u, v)(t)+(\psi(\lambda)-1) e(t)
\end{aligned}
$$

and

$$
\begin{aligned}
& D_{0^{+}}^{\beta} A\left(\lambda u+(\lambda-1) e, \lambda^{-1} v+\left(\lambda^{-1}-1\right) e\right)(t) \\
& \quad=\int_{0}^{1} D_{0^{+}}^{\beta} G(t, s) f\left(s, \lambda u(s)+(\lambda-1) e, D_{0^{+}}^{\beta}\left(\lambda^{-1} v(s)+\left(\lambda^{-1}-1\right) e\right)\right) d s-D_{0^{+}}^{\beta} e(t) \\
& \quad \geq \psi(\lambda) \int_{0}^{1} D_{0^{+}}^{\beta} G(t, s) f\left(s, u(s), D_{0^{+}}^{\beta} v(s)\right) d s-D_{0^{+}}^{\beta} e(t)+\psi(\lambda) D_{0^{+}}^{\beta} e(t)-\psi(\lambda) D_{0^{+}}^{\beta} e(t) \\
& \quad=\psi(\lambda) D_{0^{+}}^{\beta} A(u, v)(t)+(\psi(\lambda)-1) D_{0^{+}}^{\beta} e(t) .
\end{aligned}
$$

Hence, $A\left(\lambda x+(\lambda-1) e, \lambda^{-1} y+\left(\lambda^{-1}-1\right) e\right) \geq \psi(\lambda) A(x, y)+(\psi(\lambda)-1) e$. It follows from conditions (B3) and (B5) that

$$
\begin{aligned}
& H(\lambda u+(\lambda-1) e) \geq \lambda(H u)+(\lambda-1) e \\
& H\left(\lambda^{-1} u+\left(\lambda^{-1}-1\right) e\right) \leq \lambda^{-1}(H u)+\left(\lambda^{-1}-1\right) e \\
& B\left(\lambda u+(\lambda-1) e, \lambda^{-1} v+\left(\lambda^{-1}-1\right) e\right)(t) \\
& \quad=\int_{0}^{1} G(t, s) g\left(s, \lambda u(s)+(\lambda-1) e(s), H\left(\lambda^{-1} v(s)+\left(\lambda^{-1}-1\right) e(s)\right)\right) d s-e(t) \\
& \quad \geq \int_{0}^{1} G(t, s) g\left(s, \lambda u(s)+(\lambda-1) e(s), \lambda^{-1}(H v)(s)+\left(\lambda^{-1}-1\right) e(s)\right) d s-e(t) \\
& \quad \geq \lambda \int_{0}^{1} G(t, s) g(s, u(s),(H v)(s)) d s-e(t)+\lambda e(t)-\lambda e(t) \\
& \quad=\lambda B(u, v)(t)+(\lambda-1) e(t)
\end{aligned}
$$

and

$$
\begin{aligned}
& D_{0^{+}}^{\beta} B\left(\lambda u+(\lambda-1) e, \lambda^{-1} v+\left(\lambda^{-1}-1\right) e\right)(t) \\
& \quad=\int_{0}^{1} D_{0^{+}}^{\beta} G(t, s) g\left(s, \lambda u(s)+(\lambda-1) e(s), H\left(\lambda^{-1} v(s)+\left(\lambda^{-1}-1\right) e(s)\right)\right) d s-D_{0^{+}}^{\beta} e(t) \\
& \quad \geq \int_{0}^{1} D_{0^{+}}^{\beta} G(t, s) g\left(s, \lambda u(s)+(\lambda-1) e(s), \lambda^{-1}(H v)(s)+\left(\lambda^{-1}-1\right) e(s)\right) d s-D_{0^{+}}^{\beta} e(t) \\
& \quad \geq \lambda \int_{0}^{1} D_{0^{+}}^{\beta} G(t, s) g(s, u(s),(H v)(s)) d s-D_{0^{+}}^{\beta} e(t)+\lambda D_{0^{+}}^{\beta} e(t)-\lambda D_{0^{+}}^{\beta} e(t) \\
& \quad=\lambda D_{0^{+}}^{\beta} B(u, v)(t)+(\lambda-1) D_{0^{+}}^{\beta} e(t) .
\end{aligned}
$$


Thus, $B\left(\lambda u+(\lambda-1) e, \lambda^{-1} v+\left(\lambda^{-1}-1\right) e\right) \geq \lambda B(u, v)+(\lambda-1) e$. Moreover, by condition (B3)(c), we have

$$
\begin{aligned}
C\left(\lambda^{-1} v+\left(\lambda^{-1}-1\right) e\right)(t) & =\frac{\Gamma(\alpha-\gamma)}{\Gamma(\alpha)} k\left(\lambda^{-1} v+\left(\lambda^{-1}-1\right) e\right)(1) t^{\alpha-1} \\
& \geq \lambda \frac{\Gamma(\alpha-\gamma)}{\Gamma(\alpha)} k(v)(1) t^{\alpha-1}=\lambda C(v)(t)
\end{aligned}
$$

and

$$
\begin{aligned}
D_{0^{+}}^{\beta} C\left(\lambda^{-1} v+\left(\lambda^{-1}-1\right) e\right)(t) & =\frac{\Gamma(\alpha-\gamma)}{\Gamma(\alpha-\beta)} k\left(\lambda^{-1} v+\left(\lambda^{-1}-1\right) e\right)(1) t^{\alpha-\beta-1} \\
& \geq \lambda \frac{\Gamma(\alpha-\gamma)}{\Gamma(\alpha-\beta)} k(v)(1) t^{\alpha-\beta-1}=D_{0^{+}}^{\beta} \lambda C(v)(t)
\end{aligned}
$$

Thus, $C\left(\lambda^{-1} v+\left(\lambda^{-1}-1\right) e\right) \geq \lambda C(v)$. Consequently, conditions (A1)-(A3) of Theorem 2.1 are satisfied.

(3) By condition (B4), for every $u, v \in P_{h, e}, t \in[0,1]$, we have

$$
\begin{aligned}
A(u, v)(t) & =\int_{0}^{1} G(t, s) f\left(s, u(s), D_{0^{+}}^{\beta} v(s)\right) d s-e(t) \\
& \geq \delta_{1} \int_{0}^{1} G(t, s) g(s, u(s), 0) d s-e(t)-\delta_{1} e(t)+\delta_{1} e(t) \\
& \geq \delta_{1}\left(\int_{0}^{1} G(t, s) g(s, u(s),(H v)(s)) d s-e(t)\right)+(\delta-1) e(t) \\
& =\delta_{1} B(u, v)(t)+\left(\delta_{1}-1\right) e(t)
\end{aligned}
$$

and

$$
\begin{aligned}
D_{0^{+}}^{\beta} A(u, v)(t) & =\int_{0}^{1} D_{0^{+}}^{\beta} G(t, s) f\left(s, u(s), D_{0^{+}}^{\beta} v(s)\right) d s-D_{0^{+}}^{\beta} e(t) \\
& \geq \delta_{1} \int_{0}^{1} D_{0^{+}}^{\beta} G(t, s) g(s, u(s), 0) d s-D_{0^{+}}^{\beta} e(t)-\delta_{1} D_{0^{+}}^{\beta} e(t)+\delta_{1} D_{0^{+}}^{\beta} e(t) \\
& \geq \delta_{1}\left(\int_{0}^{1} D_{0^{+}}^{\beta} G(t, s) g(s, u(s),(H v)(s)) d s-D_{0^{+}}^{\beta} e(t)\right)+\left(\delta_{1}-1\right) D_{0^{+}}^{\beta} e(t) \\
& =\delta_{1} D_{0^{+}}^{\beta} B(u, v)(t)+\left(\delta_{1}-1\right) D_{0^{+}}^{\beta} e(t) .
\end{aligned}
$$

Thus, $A(u, v) \geq \delta_{1} B(u, v)+\left(\delta_{1}-1\right) e$. Similarly, we get

$$
\begin{aligned}
A(u, v)(t) & =\int_{0}^{1} G(t, s) f\left(s, u(s), D_{0^{+}}^{\beta} v(s)\right) d s-e(t) \\
& \geq \int_{0}^{1} G(t, s) \delta_{2} k(v(1)) d s-e(t) \\
& \geq \delta_{2} k(v(1)) \frac{t^{\alpha-1}}{\Gamma(\alpha)}\left(\frac{1}{\alpha-\gamma}-\frac{1}{\alpha-\beta}\right)-e(t) \\
& =\frac{\delta_{2}}{\Gamma(\alpha-\gamma)}\left(\frac{1}{\alpha-\gamma}-\frac{1}{\alpha-\beta}\right) C(v)(t)-e(t)
\end{aligned}
$$


and

$$
\begin{aligned}
D_{0^{+}}^{\beta} A(u, v)(t) & =\int_{0}^{1} D_{0^{+}}^{\beta} G(t, s) f\left(s, u(s), D_{0^{+}}^{\beta} \nu(s)\right) d s-D_{0^{+}}^{\beta} e(t) \\
& \geq \int_{0}^{1} D_{0^{+}}^{\beta} G(t, s) \delta_{2} k(v(1)) d s-D_{0^{+}}^{\beta} e(t) \\
& \geq \delta_{2} k(v(1)) \frac{t^{\alpha-\beta-1}}{\Gamma(\alpha-\beta)}\left(\frac{1}{\alpha-\gamma}-\frac{1}{\alpha-\beta}\right)-D_{0^{+}}^{\beta} e(t) \\
& =\frac{\delta_{2}}{\Gamma(\alpha-\gamma)}\left(\frac{1}{\alpha-\gamma}-\frac{1}{\alpha-\beta}\right) D_{0^{+}}^{\beta} C(v)(t)-D_{0^{+}}^{\beta} e(t) .
\end{aligned}
$$

Let $\delta_{3}=\frac{\delta_{2}}{\Gamma(\alpha-\gamma)}\left(\frac{1}{\alpha-\gamma}-\frac{1}{\alpha-\beta}\right)$, then $A(u, v) \geq \delta_{3} C(v)-e$. Choose $\delta_{4}=\min \left\{\delta_{1}, \delta_{3}\right\}$ and $\delta_{5}=\frac{1}{2} \delta_{4}$, then

$$
A(u, v) \geq \delta_{5}(B(u, v)+C(v))+\left(\delta_{5}-1\right) e .
$$

Therefore, condition (A5) of Theorem 2.1 is satisfied.

(4) Finally, we prove that condition (A4) is satisfied. By (3.1) and equality $D_{0^{+}}^{\alpha} t^{\beta}=$ $\frac{\Gamma(\beta+1)}{\Gamma(\beta-\alpha+1)} t^{\beta-\alpha}$, we have

$$
D_{0^{+}}^{\beta} h(t)=D_{0^{+}}^{\beta}\left(L t^{\alpha-1}\right)=L \frac{\Gamma(\alpha)}{\Gamma(\alpha-\beta)} t^{\alpha-\beta-1} .
$$

In view of (B1), (B2), (B4), and Lemma 2.3, we have

$$
\begin{aligned}
A(h, h)(t)+e(t) & =\int_{0}^{1} G(t, s) f\left(s, h(s), D_{0^{+}}^{\beta} h(s)\right) d s \\
& \geq \int_{0}^{1} \frac{\left[1-(1-s)^{\gamma}\right](1-s)^{\alpha-\gamma-1}}{\Gamma(\alpha)} t^{\alpha-1} f\left(s, L s^{\alpha-1}, D_{0^{+}}^{\beta} L s^{\alpha-1}\right) d s \\
& \geq \int_{0}^{1} \frac{\left[1-(1-s)^{\gamma}\right](1-s)^{\alpha-\gamma-1}}{\Gamma(\alpha)} t^{\alpha-1} f\left(s, 0, L \frac{\Gamma(\alpha)}{\Gamma(\alpha-\beta)} s^{\alpha-\beta-1}\right) d s \\
& \geq h(t) \int_{0}^{1} \frac{\left[1-(1-s)^{\gamma}\right](1-s)^{\alpha-\gamma-1}}{L \Gamma(\alpha)} f\left(s, 0, L \frac{\Gamma(\alpha)}{\Gamma(\alpha-\beta)}\right) d s \\
& \geq h(t) \int_{0}^{1} \frac{\left[1-(1-s)^{\gamma-\beta}\right](1-s)^{\alpha-\gamma-1}}{L \Gamma(\alpha)} f\left(s, 0, L \frac{\Gamma(\alpha)}{\Gamma(\alpha-\beta)}\right) d s, \\
A(h, h)(t)+e(t) & =\int_{0}^{1} G(t, s) f\left(s, h(s), D_{0^{+}}^{\beta} h(s)\right) d s \\
& \leq \int_{0}^{1} \frac{(1-s)^{\alpha-\gamma-1}}{\Gamma(\alpha)} t^{\alpha-1} f\left(s, L s^{\alpha-1}, 0\right) d s \\
& \leq h(t) \frac{1}{L \Gamma(\alpha)} \int_{0}^{1}(1-s)^{\alpha-\gamma-1} f(s, L, 0) d s,
\end{aligned}
$$

and

$$
\begin{aligned}
& D_{0^{+}}^{\beta} A(h, h)(t)+D_{0^{+}}^{\beta} e(t) \\
& \quad=\int_{0}^{1} D_{0^{+}}^{\beta} G(t, s) f\left(s, h(s), D_{0^{+}}^{\beta} h(s)\right) d s
\end{aligned}
$$




$$
\begin{aligned}
& \geq \int_{0}^{1} \frac{\left[1-(1-s)^{\gamma-\beta}\right](1-s)^{\alpha-\gamma-1}}{\Gamma(\alpha-\beta)} t^{\alpha-\beta-1} f\left(s, L s^{\alpha-1}, D_{0^{+}}^{\beta} L s^{\alpha-1}\right) d s \\
& \geq \int_{0}^{1} \frac{\left[1-(1-s)^{\gamma-\beta}\right](1-s)^{\alpha-\gamma-1}}{\Gamma(\alpha-\beta)} t^{\alpha-\beta-1} f\left(s, 0, L \frac{\Gamma(\alpha)}{\Gamma(\alpha-\beta)} s^{\alpha-\beta-1}\right) d s \\
& \geq D_{0^{+}}^{\beta} h(t) \int_{0}^{1} \frac{\left[1-(1-s)^{\gamma-\beta}\right](1-s)^{\alpha-\gamma-1}}{L \Gamma(\alpha)} f\left(s, 0, L \frac{\Gamma(\alpha)}{\Gamma(\alpha-\beta)}\right) d s, \\
D_{0^{+}}^{\beta} & A(h, h)(t)+D_{0^{+}}^{\beta} e(t) \\
& =\int_{0}^{1} D_{0^{+}}^{\beta} G(t, s) f\left(s, h(s), D_{0^{+}}^{\beta} h(s)\right) d s \\
& \leq \int_{0}^{1} \frac{(1-s)^{\alpha-\gamma-1}}{\Gamma(\alpha-\beta)} t^{\alpha-\beta-1} f\left(s, L s^{\alpha-1}, 0\right) d s \\
& \leq D_{0^{+}}^{\beta} h(t) \frac{1}{L \Gamma(\alpha)} \int_{0}^{1}(1-s)^{\alpha-\gamma-1} f(s, L, 0) d s .
\end{aligned}
$$

Let

$$
\begin{aligned}
& l_{1}=\frac{1}{L \Gamma(\alpha)} \int_{0}^{1}\left[1-(1-s)^{\gamma-\beta}\right](1-s)^{\alpha-\gamma-1} f\left(s, 0, L \frac{\Gamma(\alpha)}{\Gamma(\alpha-\beta)}\right) d s, \\
& l_{2}=\frac{1}{L \Gamma(\alpha)} \int_{0}^{1}(1-s)^{\alpha-\gamma-1} f(s, L, 0) d s .
\end{aligned}
$$

Then $l_{1} h \leq A(h, h)+e \leq l_{2} h$. Thus, $A(h, h)+e \in P_{h}$, that is, $A(h, h) \in P_{h, e}$.

On the other hand, we have

$$
\begin{aligned}
B(h, h)(t)+e(t) & =\int_{0}^{1} G(t, s) g(s, h(s),(H h)(s)) d s \\
& \geq \int_{0}^{1} \frac{\left[1-(1-s)^{\gamma}\right](1-s)^{\alpha-\gamma-1}}{\Gamma(\alpha)} t^{\alpha-1} g\left(s, 0, H\left(L s^{\alpha-1}\right)\right) d s \\
& \geq \int_{0}^{1} \frac{\left[1-(1-s)^{\gamma}\right](1-s)^{\alpha-\gamma-1}}{\Gamma(\alpha)} t^{\alpha-1} g(s, 0, H(L)) d s \\
& =h(t) \frac{1}{L \Gamma(\alpha)} \int_{0}^{1}\left[1-(1-s)^{\gamma}\right](1-s)^{\alpha-\gamma-1} g(s, 0, H(L)) d s \\
& \geq h(t) \frac{1}{L \Gamma(\alpha)} \int_{0}^{1}\left[1-(1-s)^{\gamma-\beta}\right](1-s)^{\alpha-\gamma-1} g(s, 0, H(L)) d s, \\
B(h, h)(t)+e(t) & =\int_{0}^{1} G(t, s) g(s, h(s),(H h)(s)) d s \\
& \leq \int_{0}^{1} \frac{(1-s)^{\alpha-\gamma-1}}{\Gamma(\alpha)} t^{\alpha-1} g\left(s, L s^{\alpha-1}, 0\right) d s \\
& \leq h(t) \frac{1}{L \Gamma(\alpha)} \int_{0}^{1}(1-s)^{\alpha-\gamma-1} g(s, L, 0) d s,
\end{aligned}
$$

and

$$
\begin{aligned}
& D_{0^{+}}^{\beta} B(h, h)(t)+D_{0^{+}}^{\beta} e(t) \\
& \quad=\int_{0}^{1} D_{0^{+}}^{\beta} G(t, s) g(s, h(s),(H h)(s)) d s
\end{aligned}
$$




$$
\begin{aligned}
& \geq \int_{0}^{1} \frac{\left[1-(1-s)^{\gamma-\beta}\right](1-s)^{\alpha-\gamma-1}}{\Gamma(\alpha-\beta)} t^{\alpha-\beta-1} g\left(s, L s^{\alpha-1}, H\left(L s^{\alpha-1}\right)\right) d s \\
& \geq D_{0^{+}}^{\beta} h(t) \frac{1}{L \Gamma(\alpha)} \int_{0}^{1}\left[1-(1-s)^{\gamma-\beta}\right](1-s)^{\alpha-\gamma-1} g(s, 0, H(L)) d s, \\
& D_{0^{+}}^{\beta} B(h, h)(t)+D_{0^{+}}^{\beta} e(t) \\
& \quad=\int_{0}^{1} D_{0^{+}}^{\beta} G(t, s) g(s, h(s),(H h)(s)) d s \\
& \quad \leq \int_{0}^{1} \frac{(1-s)^{\alpha-\gamma-1}}{\Gamma(\alpha-\beta)} t^{\alpha-\beta-1} g\left(s, L s^{\alpha-1}, 0\right) d s \\
& \quad \leq D_{0^{+}}^{\beta} h(t) \frac{1}{L \Gamma(\alpha)} \int_{0}^{1}(1-s)^{\alpha-\gamma-1} g(s, L, 0) d s .
\end{aligned}
$$

Let

$$
\begin{aligned}
& l_{3}=\frac{1}{L \Gamma(\alpha)} \int_{0}^{1}(1-s)^{\alpha-\gamma-1} g(s, L, 0) d s \\
& l_{4}=\frac{1}{L \Gamma(\alpha)} \int_{0}^{1}\left[1-(1-s)^{\gamma-\beta}\right](1-s)^{\alpha-\gamma-1} g(s, 0, H(L)) d s .
\end{aligned}
$$

Then $l_{4} h \leq B(h, h)+e \leq l_{3} h$, thus $B(h, h) \in P_{h, e}$. In addition, we have

$$
C(h)(t)=\frac{\Gamma(\alpha-\gamma)}{\Gamma(\alpha)} k(h(1)) t^{\alpha-1}=\frac{\Gamma(\alpha-\gamma)}{\Gamma(\alpha)} k(L) t^{\alpha-1} .
$$

Then $C(h) \in P_{h}$. Consequently, (A4) is proved. Therefore, all the conditions of Theorem 2.1 are satisfied. The conclusions of Theorem 3.1 hold.

Now, we give an example to illustrate our main result.

Example 3.1 For problem (1.1), we choose $n=5, \alpha=\frac{9}{2}, \beta=\frac{3}{2}, \gamma=\frac{5}{2}$, and $b=2$. Consider the following boundary value problem:

$$
\left\{\begin{array}{l}
D_{0^{+}}^{\frac{9}{2}} u(t)+\left[\frac{e(t)}{e^{*}} u(t)+e(t)\right]^{\frac{1}{2}}+\left[\frac{e(t)}{e^{*}} D_{0^{+}}^{\frac{3}{2}} u(t)+e(t)+1\right]^{-\frac{1}{2}} \\
\quad+\left[\int_{0}^{t}\left(u(s)+e^{*}\right) d s+e(t)+2\right]^{-\frac{1}{3}}-2=0, \quad t \in(0,1) \\
u(0)=u^{\prime}(0)=u^{\prime \prime}(0)=u^{\prime \prime \prime}(0)=0 \\
\quad\left[D_{0^{+}}^{\frac{5}{2}} u(t)\right]_{t=1}=\frac{1}{\sqrt[3]{u(1)+2}}
\end{array}\right.
$$

where $e(t)=\frac{t^{\frac{7}{2}}-\frac{4}{9} t^{\frac{9}{2}}}{\Gamma\left(\frac{9}{2}\right)}, t \in[0,1]$, and $e^{*}=\max \{e(t): t \in[0,1]\}=\frac{5}{9 \Gamma\left(\frac{9}{2}\right)}$. Clearly, $e(t) \leq \frac{t^{\frac{7}{2}}}{\Gamma\left(\frac{9}{2}\right)} \leq$ $h(t)$. Let $f(t, u, v)=\left[\frac{e(t)}{e^{*}} u+e(t)\right]^{\frac{1}{2}}+\left[\frac{e(t)}{e^{*}} v+e(t)+1\right]^{-\frac{1}{2}}, g(t, u, v)=[v+e(t)+2]^{-\frac{1}{3}}, k(u(1))=$ $\frac{1}{\sqrt[3]{u(1)+2}}$, and $(H u)(t)=\int_{0}^{t}\left(u(s)+e^{*}\right) d s$. It is easy to check that all the conditions in Theorem 3.1 are satisfied. By Theorem 3.1, problem (3.7) has a unique nontrivial solution $u^{*}$ in 
$P_{h, e}$. Furthermore, we can set up the following sequences:

$$
\begin{aligned}
\omega_{n}(t)= & \int_{0}^{1} G(t, s)\left\{\left[\frac{e(s)}{e^{*}} \omega_{n-1}(s)+e(s)\right]^{\frac{1}{2}}+\left[\frac{e(s)}{e^{*}} D_{0^{+}}^{\frac{3}{2}} \tau_{n-1}(s)+e(s)+1\right]^{-\frac{1}{2}}\right. \\
& +\left[\int_{0}^{t}\left(\tau_{n-1}(s)+e^{*}\right) d s+e(s)+2\right]^{-\frac{1}{3}}+\frac{\Gamma(2)}{\Gamma\left(\frac{9}{2}\right)} \frac{1}{\sqrt[3]{\tau_{n-1}(1)+2}} t^{\frac{7}{2}} \\
& \left.-\frac{1}{\Gamma\left(\frac{9}{2}\right)} t^{\frac{7}{2}}+\frac{4}{9 \Gamma\left(\frac{9}{2}\right)} t^{\frac{9}{2}}\right\} d s
\end{aligned}
$$

and

$$
\begin{aligned}
\tau_{n}(t)= & \int_{0}^{1} G(t, s)\left\{\left[\frac{e(s)}{e^{*}} \tau_{n-1}(s)+e(s)\right]^{\frac{1}{2}}+\left[\frac{e(s)}{e^{*}} D_{0^{+}}^{\frac{3}{2}} \omega_{n-1}(s)+e(s)+1\right]^{-\frac{1}{2}}\right. \\
& +\left[\int_{0}^{t}\left(\omega_{n-1}(s)+e^{*}\right) d s+e(s)+2\right]^{-\frac{1}{3}}+\frac{\Gamma(2)}{\Gamma\left(\frac{9}{2}\right)} \frac{1}{\sqrt[3]{\omega_{n-1}(1)+2}} t^{\frac{7}{2}} \\
& \left.-\frac{1}{\Gamma\left(\frac{9}{2}\right)} t^{\frac{7}{2}}+\frac{4}{9 \Gamma\left(\frac{9}{2}\right)} t^{\frac{9}{2}}\right\} d s,
\end{aligned}
$$

for any given $\omega_{0}, \tau_{0} \in P_{h, e}$, we have $\left\{\omega_{n}(t)\right\}$ and $\left\{\tau_{n}(t)\right\}$ both converge to $u^{*}(t)$ uniformly for all $t \in[0,1]$.

\section{Conclusions}

In this paper, we establish the existence and uniqueness theorem of a solution for the operator equation $A(x, x)+B(x, x)+C(x)+e=x$, where $A$ and $B$ are both mixed monotone, $C(x)$ is decreasing, and $e \in P$ with $P$ is a cone in Banach space $E$. Using the abstract result, we give some sufficient conditions under which problem (1.1) has a unique solution. Furthermore, we also construct two iterative sequences for approximating the unique solution.

\section{Acknowledgements}

Not applicable.

Funding

This project is supported by the Programs for the Cultivation of Young Scientific Research Personnel of Higher Education Institutions in Shanxi Province, the Scientific and Technological Innovation Programs of Higher Education Institutions in Shanxi (201802085), the Innovative Research Team of North University of China(TD201901), the Natural Science Foundation of Shanxi Province(201801D121027, 201701D221121), and the Fund for Shanxi '1331KIRT'.

\section{Availability of data and materials}

Data sharing not applicable to this article as no data sets were generated or analyzed during the current study.

Competing interests

The authors declare that they have no competing interests.

\section{Authors' contributions}

All authors contributed equally to this work. All authors read and approved the final manuscript.

\section{Author details}

'Department of Mathematics, School of Science, North University of China, Taiyuan, Shanxi, 030051, P.R. China. ${ }^{2}$ School of Information Management, Shanxi University of Finance and Economics, Taiyuan, Shanxi, 030006, P.R. China. ${ }^{3}$ School of Economics, Waseda University, 1-6-1 Nishiwaseda, Shinjuku-ku, Tokyo, 169-8050, Japan. 


\section{Publisher's Note}

Springer Nature remains neutral with regard to jurisdictional claims in published maps and institutional affiliations.

Received: 20 July 2020 Accepted: 23 February 2021 Published online: 04 March 2021

\section{References}

1. Samko, S.G., Kilbas, A.A., Marichev, O.I.: Fractional Integrals and Derivatives: Theory and Applications. Gordon \& Breach, New York (1993)

2. Kilbas, A., Srivastava, H., Trujillo, J.J.: Theory and Applications of Fractional Differential Equations. North-Holland Mathematics Studies. Elsevier, Amsterdam (2006)

3. Podlubny, I.: Fractional Differential Equations, Mathematics in Science and Engineering. Academic Press, New York (1999)

4. Afshari, H., Marasi, H., Aydi, H.: Existence and uniqueness of positive solutions for boundary value problems of fractional differential equations. Filomat 31(9), 2675-2682 (2017)

5. Liu, X.Q., Liu, L.S., Wu, Y.H.: Existence of positive solutions for a singular nonlinear fractional differential equation with integral boundary conditions involving fractional derivatives. Bound. Value Probl. 2018, 24 (2018)

6. Goodrich, C.S.: New Harnack inequalities and existence theorems for radially symmetric solutions of elliptic PDEs with sign changing or vanishing Green's function. J. Differ. Equ. 264, 236-262 (2018)

7. Goodrich, C.S.: Coercive nonlocal elements in fractional differential equations. Positivity 21, 377-394 (2017)

8. Infante, G.: Nonzero positive solutions of a multi-parameter elliptic system with functional BCs. Topol. Methods Nonlinear Anal. 52, 665-675 (2018)

9. Zhao, K.H., Gong, P.: Existence of positive solutions for a class of higher-order Caputo fractional differential equation. Qual. Theory Dyn. Syst. 14, 157-171 (2015)

10. Wang, H., Zhang, L.L., Wang, X.Q.: Fixed point theorems for a class of nonlinear sum-type operators and application in a fractional differential equation. Bound. Value Probl. 2018, 140 (2018)

11. Ji, Y.D., Guo, Y.P., Qiu, J.Q., Yang, L.Y.: Existence of positive solutions for a boundary value problem of nonlinear fractional differential equations. Adv. Differ. Equ. 2015, 13 (2015)

12. Yue, Z.Z., Zou, Y.M.: New uniqueness results for fractional differential equation with dependence on the first order derivative. Adv. Differ. Equ. 2019, 38 (2019)

13. Graef, J.R., Yang, B.: Positive solutions of a nonlinear fourth order boundary value problem. Commun. Appl. Nonlinear Anal. 14,61-73 (2007)

14. Goodrich, C.S.: Existence of a positive solution to a class of fractional differential equations. Appl. Math. Lett. 23, 1050-1055 (2010)

15. Xu, J.F., Wei, Z.L., Dong, W.: Uniqueness of positive solutions for a class of fractional boundary value problems. Appl. Math. Lett. 25, 590-593 (2012)

16. Jleli, M., Samet, B.: Existence of positive solutions to an arbitrary order fractional differential equation via a mixed monotone operator method. Nonlinear Anal., Model. Control 20(3), 367-376 (2015)

17. Yang, L., Shen, C.F., Xie, D.P.: Multiple positive solutions for nonlinear boundary value problem of fractional order differential equation with the Riemann-Liouville derivative. Adv. Differ. Equ. 2014, 284 (2014)

18. Wang, H., Zhang, L.L.: The solution for a class of sum operator equation and its applications to fractional differential equation boundary value problems. Bound. Value Probl. 2015, 203 (2015)

19. Zhang, L.L., Tian, H.M.: Existence and uniqueness of positive solutions for a class of nonlinear fractional differentia equations. Adv. Differ. Equ. 2017, 114 (2017)

20. Wang, T: The study on mixed monotone operator theories and solutions for several differential equations. Qufu Normal University, Qufu (2018) (Doctoral dissertation)

21. Sang, Y.B., Ren, Y.: Nonlinear sum operator equations and applications to elastic beam equation and fractional differential equation. Bound. Value Probl. 2019, 49 (2019)

22. Zhai, C.B., Wang, L.: $\phi-(h, e)$ concave operators and applications. J. Math. Anal. Appl. 454, 571-584 (2017)

23. Guo, D., Lakshmikantham, V.: Nonlinear Problems in Abstract Cones. Academic Press, Boston (1988)

24. Guo, D.: Partial Order Methods in Nonlinear Analysis. Jinan, Shandong Science and Technology Press (in Chinese) (2000)

25. Krasnoselskii, M.A.: Positive Solutions of Operator Equations. Noordoff, Groningen (1964)

26. Liu, L.S., Zhang, X.Q., Jiang, J., Wu, Y.H.: The unique solution of a class of sum mixed monotone operator equations and its application to fractional boundary value problems. J. Nonlinear Sci. Appl. 9, 2943-2958 (2016)

\section{Submit your manuscript to a SpringerOpen ${ }^{\circ}$ journal and benefit from:}

- Convenient online submission

- Rigorous peer review

- Open access: articles freely available online

- High visibility within the field

- Retaining the copyright to your article

Submit your next manuscript at $\gg$ springeropen.com 